\title{
HIPERTROFIA DEL MÚSCULO MASETERO UNILATERAL - ESTUDIO DE CASO-
}

AUTORES:

INSTITUCIÓN: PAIS:

\section{RESUMEN:}

Objetivos: registrar y analizar la actividad eléctrica de los músculos masticatorios en el caso de Hipertrofia masetérica. Métodos: paciente femenina, 34 años, presenta un aumento del ángulo izquierdo de la mandíbula, desde la adolescencia. Cefaleas constantes. En la cirugía se retiró tejido muscular masetérico y óseo mandibular. En la evaluación preoperatoria fue realizado un examen complementario de electromiografía de superficie, para registrar los promedios de las actividades eléctricas de los músculos temporales, porción anterior, y maseteros, en la situación de reposo y masticación. El examen fue repetido luego de 21 días, tres y seis meses posoperatorio (P.O.), con orientaciones fonoaudiológicas. Resultados: preoperatorio, los maseteros registraron en reposo y masticación, promedios mayores en relación a los temporales, y el masetero izquierdohipertrófico- con promedios mucho mayores en relación al derecho. A los 21 días P.O., en reposo y masticación-amasamiento-, los temporales registraron promedios mayores en comparación con los maseteros. Tres meses P.O., en reposo, los promedios de los temporales fueron cercanas a las de los maseteros, y en la masticación permanecieron promedios mayores que los de los temporales comparado a los maseteros. Seis meses P.O., en reposo, el músculo masetero izquierdo registró un promedio mayor en relación al temporal ipsilateral, y en la masticación se registró una mayor actividad del temporal en relación al masetero ipsilateral. La paciente faltó a $75 \%$ de las sesiones fonoaudiológicas propuestas. Conclusiones: la electromiografía demostró en $21^{\circ}$ día P.O. la eficacia de la cirugía, registrando mayor actividad para el músculo temporal en reposo. El promedio de la actividad mostró que seis meses P.O. los músculos masticatorios se encontraban en desarmonía en el reposo y masticación, tal vez por la ausencia de la paciente a la terapia miofuncional. La decisión del tratamiento quirúrgico favoreció la estética y la rehabilitación de los músculos y funciones. Exámenes complementares, como la electromiografía, auxiliaron en la conducta terapéutica pre y posquirúrgica y en las orientaciones fonoaudiológicas, en el área de motricidad orofacial.

Palabras clave: Hipertrofia, Músculo Masetero 